Case Report

\title{
Beckwith-Wiedemann Syndrome: A Case Report at the Gynaeco-Obstetric and Pediatric Hospital in Yaounde, Cameroon
}

\author{
Daniel Armand Kago Tague ${ }^{1, ~}$, Evelyn Mah ${ }^{1}$, Félicitee $\operatorname{Nguefack}^{1}$, Georges Pius Kamsu Moyo ${ }^{1}$, \\ Lionel Loic Kago Tcheyanou ${ }^{1}$, Faustin Mouafo $^{2}$ \\ ${ }^{1}$ Department of Paediatrics, Faculty of Medicine and Biomedical Sciences, the University of Yaounde I, Yaounde, Cameroon \\ ${ }^{2}$ Department of Surgery, Faculty of Medicine and Biomedical Sciences, the University of Yaounde I, Yaounde, Cameroon
}

\author{
Email address: \\ daniel.kago@gmail.com (D. A. K. Tague) \\ *Corresponding author
}

\section{To cite this article:}

Daniel Armand Kago Tague, Evelyn Mah, Félicitee Nguefack, Georges Pius Kamsu Moyo, Lionel Loic Kago Tcheyanou, Faustin Mouafo. Beckwith-Wiedemann Syndrome: A Case Report at the Gynaeco-Obstetric and Pediatric Hospital in Yaounde, Cameroon. American Journal of Pediatrics. Vol. 6, No. 4, 2020, pp. 433-436. doi: 10.11648/j.ajp.20200604.17

Received: September 28, 2020; Accepted: October 15, 2020; Published: October 23, 2020

\begin{abstract}
Beckwith-Wiedemann syndrome is a rare congenital syndrome, but one of the most common among overgrowth syndromes. It was described firstly by Beckwith in 1963. The incidence of BWS is about 1:13 700 births, with an equal sex distribution. It is characterized by macrosomia, macroglossia, omphalocele and anterior abdominal wall defects. It is a complex multigenic disorder caused by dysregulation of the gene printed on chromosome 11p. 15. A relationship has been established between assisted fertilization methods and its occurrence. This syndrome predisposes to childhood cancer. Its incidence is not known in developing countries such as Cameroon due to the rarity of reported cases, variability in the presentation of the syndrome, financial constraints and lack of access to genetic and molecular studies. In our opinion, we are reporting the first case of Beckwith-Wiedemann syndrome in our country. We present here the case of a newborn female baby delivered at the gyneco-obstetric and pediatric hospital in Yaoundé and admitted to our neonatology unit. The diagnosis was made on the basis of clinical signs suggestive of Beckwith-Wiedemann syndrome. She presented an omphalocele diagnosed on antenatal ultrasound, macrosomia, macroglossia and ear abnormalities. The case is presented to raise awareness and highlight the particularity of the management of this rare disease.
\end{abstract}

Keywords: Beckwith-Wiedemann, Macrosomia, Macroglossia, Omphalocele

\section{Introduction}

Beckwith-Wiedemann syndrome (BWS) is a human imprinting disorder that leads to overgrowth [1]. This syndrome was described independently by two investigators; Beckwith in 1963 and Wiedemann in 1964 [2, 3]. BWS is clinically and genetically a heterogeneous disorder and it has an estimated incidence of one in 13,700 [4-6].

BWS involves molecular aberrations within a cluster of imprinted genes on the chromosome 11p15.5-11p15.4 region [7]. About $80 \%$ of patients with BWS have a known moleculardefect in the $11 \mathrm{p} 15$ region, most commonly due to aberrant DNAmethylation [8]. Children conceived through In vitro fertilization have a three to four fold increased chance of developing BWS. It is thought that this is due to genes being turned on or off by the IVF procedures [7].

The baby with BWS usualy presents with the combination of congenital abdominal wall defects as hernia (exomphalos), large tongues (macroglossia), and large bodies and/or long limbs (gigantism). In addition, some children with BWS have other findings including: nevus flammeus, prominent occiput, midface hypoplasia, hemihypertrophy, genitourinary anomalies (enlarged kidneys), cardiac anomalies, musculoskeletal abnormalities, and hearing loss. Also, some 
premature newborns with BWS do not have macroglossia until closer to their anticipated delivery date [9].

Another definition presented by Elliot et al. includes the presence of either three major features (anterior abdominal wall defect, macroglossia, or prepostnatal overgrowth) or two major plus three minor findings (ear pits, nevus flammeus, neonatal hypoglycemia, nephromegaly, or hemihyperplasia) [10]. While most children with BWS do not develop cancer, children with BWS do have a significantly increased risk of cancer [11]. Children with BWS are most at risk during early childhood and should receive cancer screening during this time $[9,12]$.

\section{Case Report}

One day old female baby, born by Yaounde Gynaeco-Obstetric and pediatric Hospital by cesarian section indicated for cicatricle uterus at a term of 35SA and 3 days. During the antennal screening we have an USG which presented omphalocele. At the birth she presented a mild respiratory distress and a small omphalocele type 2 . There was no history of birth asphyxia. No IVF. she was $3.7 \mathrm{~kg}$ and. On examination of this baby, the weight was $3.37 \mathrm{kgs}$, (macrosomia), length $45 \mathrm{cms}$, head circumference $34 \mathrm{cms}$. Cry, reflex, tone, activity was good. On head to toe examination, macroglossia (figure 1), nevus flammeus (figure 1a), ear lobe crease (figure 1b) and no hemi hypertrophy of the body, left MUAC $7 \mathrm{cms}$ comparisons to right $1 \mathrm{cms}$, left thigh $15 \mathrm{cms}$ in comparisons to right $15 \mathrm{cms}$. On investigation blood sugar was normal $50 \mathrm{mg} / \mathrm{dl}$ and child was admitted and treated mild distress and reduction of her omphalocele. Complete blood count, serum electrolyte, renal function test, liver function test, TSH and T4 were normal. On USG, liver and both kidneys were normal, echocardiography normal.

\section{Discussion}

BWS syndrome in genetic disorder is characterized by overgrowth, tumor predisposition and congenital malformation (13). Although incidence is around every 15000 births, but presentation may vary from child to child, thus difficult to diagnose (14).

Common features used to define BWS are $[9,12]$

1) Macroglossia (large tongue),

2) Macrosomia (above average birth weight and length), above $95 \%$ centile

3) Midline abdominal wall defects (omphalocele/exomphalos, umbilical hernia, diastasis recti),

4) Ear creases or ear pits,

5) Neonatal hypoglycemia (low blood sugar after birth).

6) Hepatoblastoma

This baby having three major features like omphalocele, macrosomia and macroglossia and also having minor findings like nevus flammeus, ear pits $(15,16)$.

The most common tumor associated with this syndrome are Wilms tumor $(52 \%)$, hepatoblastoma (14\%), neuroblastoma (10\%), rhabdomyosarcoma $(5 \%)$, adrenocortical carcinoma
(3\%) [7]. Most children ( $>80 \%$ ) with BWS do not develop cancer; however, children with BWS are much more likely ( $\sim 600$ times more) than other children to develop certain childhood cancers, particularly Wilms' tumour (nephroblastoma), pancreato-blastoma and hepatoblastoma $[12,17]$. Individuals with BWS appear to only be at increased risk for cancer during childhood (especially before age four) and do not have an increased risk of developing cancer in adulthood [12]. Diagnosis mainly clinical correlation along with molecular testing, molecular testing confirm the diagnosis but it does not rule out BWS. Antenatal ultrasonography (USG) can detect major abdominal defect, chorionic villous sampling and amniocentesis can detect genetics changes [7, 15]. Supportive medical care and corrective surgery is mainstay of treatment.

Neonatal hypoglycemia, low blood glucose in the first month of life, occurs in about half of children with BWS [18]. Usually this hypoglycemia can easily be treated with more frequent feedings or medical doses of glucose. Rarely $(<5 \%)$ children with BWS will continue to have hypoglycemia after the neonatal period and require more intensive treatment $[18$, 19].

For abdominal wall problem, corrective surgery required only in case of omphalocele, umbilical hernia, diastasis recti may resolve spontaneously [7].

Macroglossia in BWS becomes less noticeable with age and often requires no treatment; but it does cause problems for some children with BWS. In severe cases, macroglossia can cause respiratory, feeding, and speech difficulties may require surgery [20]. It is seen in about $90 \%$ of patients with BWS [7]. The best time to perform surgery for a large tongue is not known. Studies show that patients who receive surgery before 2-3 years tend to have good outcomes with favorable results including cosmetic improvement, adequate tongue mobility, and no substantial effect on taste [7]. Surgery for macroglossia involves removing a small part of the tongue so that it fits within the mouth to allow for proper jaw and tooth development [21].

Nevus flammeus (port-wine stain) is a flat, red birthmark caused by a capillary (small blood vessel) malformation. Children with BWS often have nevus flammeus on their forehead or the back of their neck [1, 22]. Nevus flammeus is benign and commonly does not require any treatment.

Ear Lobe Creases, these are sometimes found in conjunction with indentations behind the upper rim of the ear [22].

Wilms Tumors; tumors of the kidney. Around 7.5\% of BWS children will develop Wilms Tumor. Because of the aggressiveness of these tumors, abdominal ultrasound scans should take place every three months up to the age of 7 or 8 years. A baseline MRI scan may also be performed. The susceptibility to these tumors diminishes and is not usually a problem after the age of 8 . Please note that not all children with BWS are at risk of Wilms tumor and therefore do not require USG scan. Sometimes this will require surgery to remove the affected kidney and possibly chemotherapy and radiotherapy $[12,23]$. 
Visceromegaly, Enlarged abdominal organs, usually the kidneys, liver, spleen, adrenals and pancreas.

Hemi hypertrophy, May require orthopedic surgery though is often treated with a shoe lift. Isolated hemi hypertrophy is associated with a higher risk for cancer [24]. The types of cancer and age of the cancers are similar to children with BWS As a result, children with hemi hypertrophy should follow the general cancer screening protocol for BWS [25].

Hepatoblastoma, the risk of these diminishes after the age of 3 years. They can also be detected by abdominal ultrasound but, as not all the liver can be viewed, AFP (alpha-feta-protein) levels in the blood may also be monitored 3 monthly [17]. As the risk of these tumors is so low, this test is not usually carried out [7].

Cardiac defects occur in up to $13-20 \%$ of patients with BWS, andthere is higher incidence of congenital heart disease comparedto the general pediatric population [7]. Minor anatomical defects should be monitored by echocardiogramuntil spontaneous resolution, but more severe defects mightrequire surgical correction [15].

Prognosis

In general, the prognosis is very good. Children with BWS usually do very well and grow up to become the heights expected based on their parents' heights. While children with BWS are at increased risk of childhood cancer, most children with BWS do not develop cancer and the vast majority of children who do develop cancer can be treated successfully. Children with BWS for the most part had no significant delays when compared to their siblings. However, some children with BWS do have speech problems that could be related to macroglossia or hearing loss. Advances in treating neonatal complications and premature infants in the last twenty years have significantly improved the true infant mortality rate associated with BWS.

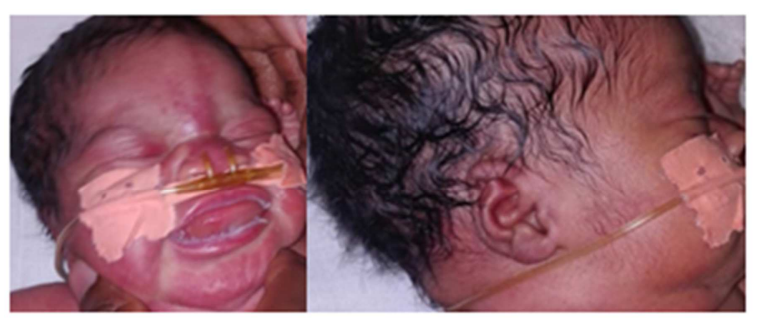

(a)

(b)

Figure 1. The clinical pictures show macroglossia, nevus flammeus ear lobe Creases.

\section{Conclusion}

Children with BWS usually do very well and grow up to become the heights expected based on their parents' heights. As children with BWS are at increased risk of childhood cancer, they should follow up strictly for cancer screening. An abdominal ultrasound every 3 months until at least eight years of age is recommended and a blood test to measure alpha-fetoprotein (AFP) every 12 weeks until at least four years of age. Families and physicians should determine screening schedules for specific patients, especially the age at which to discontinue screening, based upon their own evaluation of the risk-benefit ratio.

\section{References}

[1] Molloy SF, Kanyama C, Heyderman RS, Loyse A, Kouanfack C, Chanda D, et al. Antifungal combinations for treatment of cryptococcal meningitis in Africa. N Engl J Med. 2018; 378 (11): 1004-17.

[2] JB B. Extreme cytomegaly of the adrenal fetal cortex, omphalocele, hyperplasia of kidneys and pancreas, and Leydig-cell hyperplasia: another syndrome? Annu Meet West Soc Pediatr Res Los Angeles, California; 1963. 1963.

[3] HR. W. Familial malformation complex with umbilical hernia and Macroglossia--a "new syndrome"? J Genet Hum. 1964; 13: 223-32.

[4] Mohsin F, Islam R, Begum T, Azad K, Nahar N. Beckwith Wiedemann Syndrome. 2014; 32 (3).

[5] Cammarata-Scalisi F, Avendaño A, Stock F, Callea M, Sparago A, Ricciod A. Beckwith-Wiedemann syndrome. Clinical and etiopathogenic aspects of a model genomic imprinting entity. Vol. 116, Archivos Argentinos de Pediatria. 2018.

[6] Gupta I, Sankar D. Medical Attention at Death: Evidence from India. J Health Manag [Internet]. 2004 Apr 24 [cited 2020 Sep 8]; 6 (1): 73-84. Available from: http://journals.sagepub.com/doi/10.1177/09720634040060010 5

[7] Wang KH, Kupa J, Duffy KA, Kalish JM. Diagnosis and Management of Beckwith-Wiedemann Syndrome. Vol. 7, Frontiers in Pediatrics. 2020.

[8] Choufani S, Shuman C, Weksberg R. Beckwith-Wiedemann syndrome. Vol. 154, American Journal of Medical Genetics, Part C: Seminars in Medical Genetics. 2010.

[9] Thorburn MJ, Wright ES, Miller CG, Smith-Read EHMN. Exomphalos-Macroglossia-Gigantism Syndrome in Jamaican Infants. Am J Dis Child [Internet]. 1970 Apr 1 [cited 2020 Oct 14]; 119 (4): 316-21. Available from: https://jamanetwork.com/journals/jamapediatrics/fullarticle/50 3525 .

[10] Elliott M, Bayly R, Cole T, Temple IK ME. "Clinical features and natural history of Beckwith-Wiedemann syndrome: presentation of 74 new cases". Clin Genet. 1994; 46 (2): 168 74.

[11] Pellegrin MC, Spinelli AM, Tornese G, Barbi E. Unilateral testicular enlargement in a teenager with Beckwith-Wiedemann syndrome: A case report. Ital J Pediatr. 2019; 45 (1).

[12] DeBaun MR, Tucker MA. Risk of cancer during the first four years of life in children with The Beckwith-Wiedemann Syndrome Registry. J Pediatr. 1998 Mar 1; 132 (3 I): 398-400.

[13] Idrees H, Qureshi D, Abro B. Beckwith-Wiedemann syndrome. Pakistan Paediatr J. 2017; 41 (3).

[14] De Paoli A, Di Francesco A, Selicorni A. Beckwith-Wiedemann syndrome. Med e Bambino. 2019; 38 (8). 
[15] Brioude F, Kalish JM, Mussa A, Foster AC, Bliek J, Ferrero $\mathrm{GB}$, et al. Clinical and molecular diagnosis, screening and management of Beckwith-Wiedemann syndrome: An international consensus statement. Nat Rev Endocrinol. 2018 Apr 1; 14 (4): 229-49.

[16] Barisic I, Boban L, Akhmedzhanova D, Bergman JEH, Cavero-Carbonell C, Grinfelde I, et al. Beckwith Wiedemann syndrome: A population-based study on prevalence, prenatal diagnosis, associated anomalies and survival in Europe. Eur J Med Genet. 2018; 61 (9).

[17] Zivot A, Edelman M, Glick R, Hong A, Fish JD. Congenital Hepatoblastoma and Beckwith-Wiedemann Syndrome. J Pediatr Hematol Oncol. 2019.

[18] DeBaun MR, King AA, White N. Hypoglycemia in Beckwith-Wiedemann syndrome. Semin Perinatol. 2000 Apr 1; 24 (2): 164-71.

[19] Munns CFJ, Batch JA. Hyperinsulinism and Beckwith-Wiedemann syndrome [Internet]. Vol. 84, Archives of Disease in Childhood: Fetal and Neonatal Edition. BMJ Publishing Group; 2001 [cited 2020 Oct 14]. p. F67-9. Available from: http://fn.bmj.com/.

[20] Alonso-Rodriguez E, Gómez E, Martín M, Muñoz JM, Hernández-Godoy J, Burgueño M. Beckwith-wiedemann syndrome: Open bite evolution after tongue reduction. Vol. 23, Medicina Oral Patologia Oral y Cirugia Bucal. 2018.
[21] Naujokat H, Möller B, Terheyden H, Birkenfeld F, Caliebe D, Krause MF, et al. Tongue reduction in Beckwith-Wiedemann syndrome: outcome and treatment algorithm. Int $\mathrm{J}$ Oral Maxillofac Surg. 2019; 48 (1).

[22] Wang R, Xiao Y, Li D, Hu H, Li X, Ge T, et al. Clinical and molecular features of children with Beckwith-Wiedemann syndrome in China: A single-center retrospective cohort study. Ital J Pediatr [Internet]. 2020 Apr 29 [cited 2020 Oct 14]; 46 (1) 1-7. Available from: https://link.springer.com/articles/10.1186/s13052-020-0819-3.

[23] White JC, Liu J, Nahar A. Simultaneous Presentation of Wilms Tumor and Immature Ovarian Teratoma in Beckwith-Wiedemann Syndrome. J Pediatr Hematol Oncol. 2018; 40 (1).

[24] Vaiman M, Shilco P, Roitblat Y, Nehuliaieva L, Rosenberg S, Leit A, et al. Hemihyperplasia/hemihypertrophy in adolescents: Prospective international study. Int $\mathrm{J}$ Adolesc Med Health [Internet]. 2019 Jan 12 [cited 2020 Oct 14]; 1 (ahead-of-print). Available from: https://www.degruyter.com/view/journals/ijamh/ahead-of-prin t/article-10.1515-ijamh-2018-0066/article-10.1515-ijamh-201 8-0066.xml.

[25] Abraham P. What is the risk of cancer in a child with hemihypertrophy? Vol. 90, Archives of Disease in Childhood. 2005. p. 1312-3. 